\title{
Visual loss resulting from siderosis bulbi caused by a missed intraocular foreign body
}

\author{
Simona Delia Nicoară, Ioana Damian
}

\begin{abstract}
Introduction: Intraocular foreign bodies (IOFB) represent an important cause of visual impairment, especially within the group of working age population. Case report: A 38-yearold male presented with severe left eye pain, accompanied by left headache, visual loss, redness, photophobia, tearing and nausea, 30 months following a minor left eye trauma when hammering metal on metal. Eye ultrasound identified a small intraocular foreign body as well as the crystalline lens luxated into the vitreous cavity. Pars plana vitrectomy was carried out with the extraction of the foreign body and of the lens. Pain was relieved, but vision was not regained. Conclusion: The poor outcome of this case follows from a combination of unusual factors: ophthalmologists' failure to identify an occult IOFB and the lack of patient's concern about his eye condition, until the moment when he experienced excessive pain and vision was already lost.
\end{abstract}

Keywords: Intraocular foreign body, Pars plana vitrectomy, Siderosis bulbi

Simona Delia Nicoară ${ }^{1,2}$, Ioana Damian ${ }^{3}$

Affiliations: ${ }^{1}$ Professor, Department of Ophthalmology, "Iuliu Hațieganu" University of Medicine and Pharmacy, Cluj-Napoca, Cluj, Romania; ${ }^{2}$ Senior ophthalmologist, Department of Ophthalmology, Emergency County Hospital, Cluj-Napoca, Cluj, Romania; ${ }^{3}$ Assistant Professor, Department of Ophthalmology, "Iuliu Hațieganu" University of Medicine and Pharmacy, Cluj-Napoca, Cluj, Romania.

Corresponding Author: Simona Delia Nicoară, 8, V. BabeșStreet, Cluj-Napoca, Cluj, Romania 400012; Email: simonanicoara1@gmail.com

Received: 27 March 2018

Accepted: 01 May 2018

Published: 23 May 2018

\section{How to cite this article}

Nicoară SD, Damian I. Visual loss resulting from siderosis bulbi caused by a missed intraocular foreign body. Case Rep Int 2018;7:100047Zo6SN2018.

Article ID: 100047Zo6SN2018

$* * * * * * * * *$

doi:10.5348/100047Zo6SN2018

\section{INTRODUCTION}

Intraocular foreign bodies (IOFB) represent an important cause of visual impairment, especially within the group of working age population $[1,2,3]$. This report illustrates the case of a young male who lost vision in one eye, following a minor trauma resulting in an intraocular foreign body that was not timely recognized. The poor outcome in this case follows from an unusual combination of factors: two ophthalmologists who failed to identify an occult IOFB and a patient who was not very concerned about his eye condition, up to the moment when he experienced excessive pain. The importance of thorough clinical and imaging examination of the eye in emergency setting is highlighted and siderosis bulbi, a rare condition in current clinical practice, is commented.

\section{CASE REPORT}

A 38-year-old male presented with severe left eye pain, accompanied by left headache, visual loss, redness, photophobia, tearing and nausea.

Thirty months ago, the patient had suffered a minor left eye trauma when hammering metal on metal. Although vision was not affected, he presented himself immediately at the ophthalmology emergency room. Following clinical examination, eye drops were prescribed. Two months following trauma, the patient 


\section{EDORIUM Journals}

www.casereportsinternational.com

noticed progressive constriction of the visual field (VF) left eye (OS), followed by gradual visual loss OS, especially in darkness (nyctalopia) and iris turning rustcoloured. The patient consulted another ophthalmologist who identified atonic pupil and constriction of the VF OS, but indicated no further examination. Since that date, the patient did not consult another ophthalmologist, until the occurence of the acute episode described above.

Upon presentation, visual acuity was 20/20 right eye (OD) and counting fingers OS. The patient had left exotropia, relative afferent pupillary defect OS and iris heterochromia. OS was red (predominantly in the perilimbal area), and the cornea was hazy, with intrastromal rust-coloured deposits. The lens was absent from the pupillary area and iridodonesis was noticed. The anterior chamber was deeper in OS as compared to OD, with no sign of inflammation. Iris heterochromia is presented in Figure 1. The clinical exam of the posterior segment OS could not identify any detail, because of the hazy cornea. Gonioscopy revealed an open angle (Schaffer grade IV), with rusty pigmentation on 360 degrees, particularly in the trabecular meshwork (TM). Intraocular pressure was $48 \mathrm{mmHg}$ in OS, measured by applannation tonometry. The fellow eye was unremarkable.

Eye ultrasound T6 identifying the IOFB is presented in Figure 2. B-scan shows the IOFB as an echo-dense signal with marked shadowing posterior to it, measuring 1.9/1.1 $\mathrm{mm}$ and being fixed at the eye wall, at 6 o'clock. A-scan proves the high amplitude echo given by the IOFB, with distinct acoustic shadow. Eye ultrasound T10 shows a biconvex structure (the crystalline lens), adherent to the eye wall, located in the supero-nasal quadrant (Figure 3). A and B-scans prove the highly echogenic lens capsule, enclosing the low echogenic nucleus.

The final diagnosis is siderosis bulbi with luxated lens into the vitreous cavity and secondary glaucoma.

Fixed combination of timolol and dorzolamide bid OS was promptly initiated. Systemic carbonic anhydrase inhibitor $1.5 \mathrm{~g} /$ day was added. Despite maximum topical and systemic regimen, IOP remained elevated above $40 \mathrm{mmHg}$. Pars plana vitrectomy was carried out, with removal of the IOFB and of the crystalline lens from the vitreous cavity. The foreign body was strongly attached to the retina and required thorough dissection in order to be removed. Endolaser was applied arround the foreign body's site. Retina was attached at the end of surgery.

The next day following surgery, symptoms were significantly reduced, but vision remained very low (counting fingers). Under topical treatment with fixed combination of eye drops containing tobramycin and dexamethasone, the symptoms were completely relieved, but there was no improvement in vision. The patient failed to attend the scheduled visits, as he went to work abroad.

\section{DISCUSSION}

Time limitations which are specific to the emergency setting create the context for quick and superficial

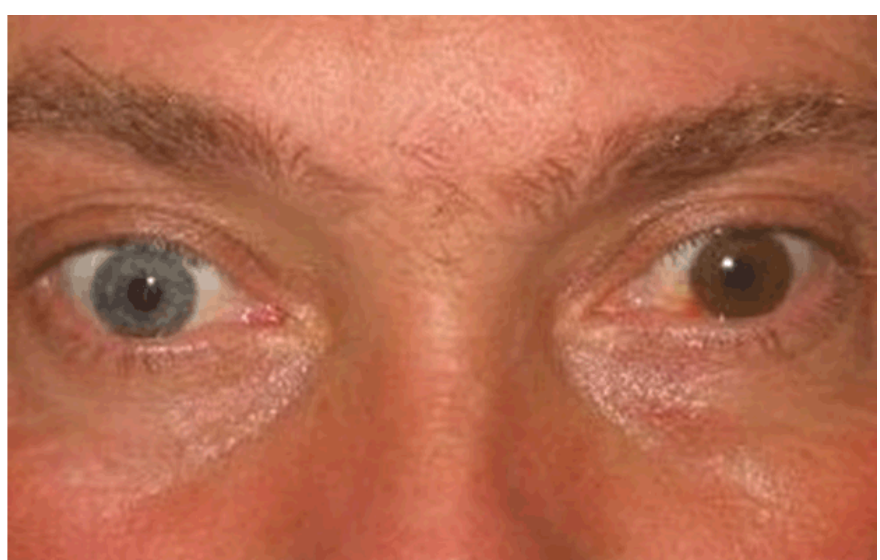

Figure 1: Iris heterochromia: left iris is brown as compared to the right iris which is blue.

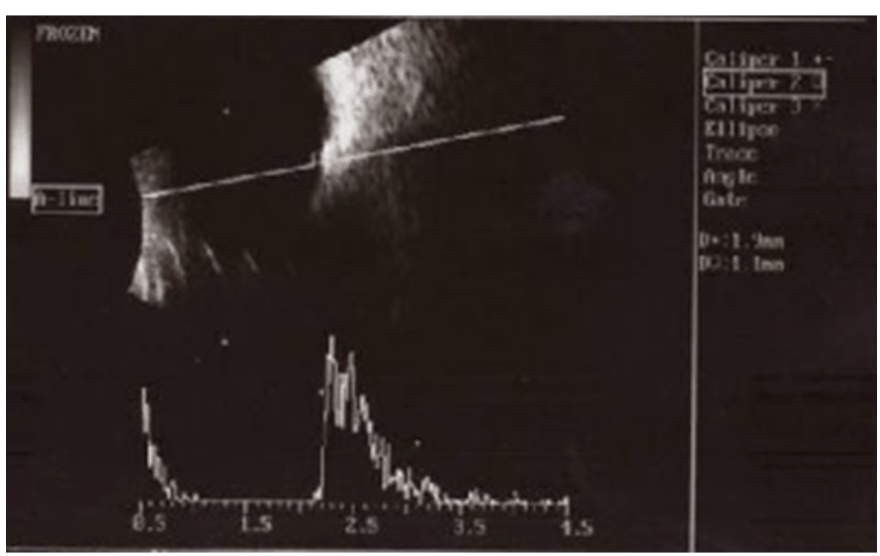

Figure 2: Eye ultrasound T6 identifying the intraocular foreign body: B-scan shows the IOFB as an echo-dense signal with marked shadowing posterior to it, measuring $1.9 / 1.1 \mathrm{~mm}$ and being fixed at the eye wall, at 6 o'clock. A-scan proves the high amplitude echo given by the IOFB, with distinct acoustic shadow.

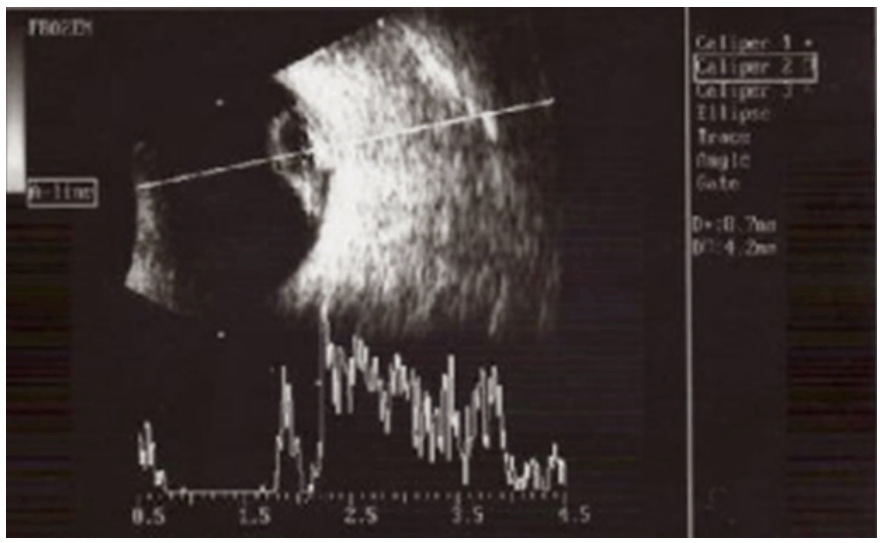

Figure 3: Eye ultrasound T10 shows a biconvex structure (the crystalline lens), adherent to the eye wall, located in the superonasal quadrant. A and B-scans prove the highly echogenic lens capsule, enclosing the low echogenic nucleus.

examination of patients. This case is strong in highlighting theimportance of thorough ophthalmological examination in a patient with suggestive history of IOFB, but no visible 


\section{EDORiUM Journals}

entry site. Its main point is that indicating in emergency a simple imaging method (CT, eye ultrasound, radiogram) would have identified the IOFB, with significant impact on patient's visual prognosis. The main limitation of this report is the relative lack of information between the moment of trauma and the actual presentation.

In most instances, a penetrating or a perforating injury of the eye is easily recognizable. However, an occult ocular penetrating injury requires a highly suspicious attitude in order to be identified, taking into account the risk of severe complications associated to it, as documented by our case [1]. In this case, the IOFB produced no significant lesion upon penetrating the eye and excited minimum inflammation. Therefore, it remained quiescent for a long period of time.

Before the actual presentation, the patient had consulted ophthalmologists twice. In emergency, no entry site in the eye was identified and other than a mild conjunctival injection, the clinical examination revealed no abnormality. However, the occurence of eye trauma while hammering metal on metal was highly suggestive for IOFB.Therefore, a CT-scan, an eye ultrasound or even a simple radiogram would have identified the IOFB, in a relatively fast and non-invasive manner. CT-scanning is the preferred primary imaging modality, being able to detect metal IOFBs with a threshold of $0.07 \mathrm{~mm}^{3}[2,3]$. During the second consultation (two months following trauma), the patient displayed typical symptoms and signs of siderosis bulbi (iris heterochromia, visual field constriction, nyctalopia, decreased visual acuity, atonic pupil) which would have led to conduct the necessary imaging investigations in order to identify the IOFB.

Even if small, the IOFB had devastating consequences on the eye, due to retinal cytotoxic reactions related to its decomposition [4]. Inside the eye, iron generates oxidants that inactivate the enzymes, leading to the damage of photoreceptor cell membranes. Ferritin released from the IOFB is deposited throughout the cytoplasm of the ocular cells in the form of siderosomes, with subsequent citotoxic effects [5]. Chao and coworkers, after having induced experimental siderosis by injecting Iron Sulfate (FeSO4) in the eyes of Wistar rats, noticed the disorganization of the retina, with loss of photoreceptor outer segments and cholinergic amacrine cells. They suggest that retinal damage is produced by the combination of oxidative stress with excessive glutamate release and increased calcium influx [6].

Siderosis bulbi may appear from 18 days up to many years after the ocular trauma [7]. Our case displayed typical clinical features of siderosis bulbi: iris heterochromia, visual field constriction, nyctalopia, fixed midriasis, siderotic cataract with lens dislocation, secondary glaucoma [7]. Secondary glaucoma in ocular siderosis is explained by several theories: the physical obstruction of the trabecular meshwork (TM) with metallic particles, fibrosclerosis of the TM and ciliary body changes $[4,7]$. In our case, the secondary glaucoma may be also phacolytic, caused by leakage of lens proteins through the hypermature capsule. TM gets obstructed by released lens material which is composed of altered lens protein, macrophages and other inflammatory cells [8].

Since there was no retinal detachment at the moment of IOFB removal, we did not inject silicone oil at the end of surgery. Even if retina was attached, visual function recovery is doubtful in this case, taking into account retinal cytotoxicity inflicted along IOFB decomposition. Siderosis induced irreversible retinal damage is the main reason why we did not consider intraocular lens implantation.

\section{CONCLUSION}

Imaging methods should be conducted in all patients with highly suggestive history of IOFB, even in the absence of a visible entry site. The failure to diagnose siderosis bulbi during the second ophthalmological exam might be explained by the rarity of this condition in current practice. Association of progressive change in iris color, visual field constriction and nyctalopia is highly suggestive for this disease, especially in posttraumatic setting.

\section{REFERENCES}

1. Yeh S, Ralle M, Phan IT, Francis PJ, Rosenbaum JT, Flaxel CJ. Occult intraocular foreign body masquerading as panuveitis: Inductively coupled mass spectrometry and electrophysiologic analysis. J Ophthalmic Inflamm Infect 2012 Jun;2(2):99-103.

2. Loporchio D, Mukkamala L, Gorukanti K, Zarbin M, Langer P, Bhagat N. Intraocular foreign bodies: A review. Surv Ophthalmol 2016 Sep-Oct;61(5):58296.

3. Nicoara SD, Irimescu I, Calinici $\mathrm{T}$, Cristian C. Intraocular foreign bodies extracted by pars plana vitrectomy: Clinical characteristics, management, outcomes and prognostic factors. BMC Ophthalmol 2015 Nov 2;15:151.

4. Ding J, Yoganathan P, Fernando-Sieminski S. Resolution of siderosis glaucoma from chronic intraocular foreign body without glaucoma surgery. Can J Ophthalmol 2015 Oct;50(5):e82-5.

5. Tawara A. Transformation and cytotoxicity of iron in siderosis bulbi. Invest Ophthalmol Vis Sci 1986 Feb;27(2):226-36.

6. Chao HM, Chen YH, Liu JH, et al. Iron-generated hydroxyl radicals kill retinal cells in vivo: Effect of ferulic acid. Hum Exp Toxicol 2008 Apr;27(4):32739.

7. Asencio-Duran M, Vázquez-Colomo PC, ArmadáMaresca F, Fonseca-Sandomingo A. Siderosis bulbi. Clinical presentation of a case of three years from onset. [Article in Spanish]. Arch Soc Esp Oftalmol 2012 Jun;87(6):182-6.

8. Kanski J, Bowling B. Lens-related glaucoma. In: Kanski J, Bowling B, editors. Clinical Ophthalmology: A Systematic Approach. 7ed. London: Elsevier Saunders; 2011. p. 364-6. 


\section{SUGGESTED READING}

- webeye.ophth.uiowa.edu/eyeforum/atlas/pages/ siderosis-bulbi/index.htm

- $\quad$ www.aao.org/image/siderosis-bulbi-5

- $\quad$ www.hindawi.com/journals/joph/2016/7272465/

- $\quad$ www.ncbi.nlm.nih.gov/pubmed/2336277

- $\quad$ www.ncbi.nlm.nih.gov/pmc/articles/PMC4616819/

- http://bjo.bmj.com/content/

bjophthalmol/38/12/727.full.pdf

- $\quad$ http://www.ajo.com/article/s0oo2-9394(98)00298o/abstract

- www.slideshare.net/laxmieyeinstitute/intraocularforeign-body

- $\quad$ http://ocw.tufts.edu/data/37/487903.pdf

- $\quad$ http://slideplayer.com/slide/3533276/

$* * * * * * * * *$

\section{Acknowledgements}

This study was funded by grant number PED 156, Executive Agency for Higher Education, Research, Development and Innovation Funding, Romania

\section{Author Contributions}

Simona Delia Nicoară - Substantial contributions to conception and design, Acquisition of data, Analysis and interpretation of data, Drafting the article, Revising it critically for important intellectual content, Final approval of the version to be published
Ioana Damian - Acquisition of data, Revising it critically for important intellectual content, Drafting the article, Final approval of the version to be published

\section{Guarantor of Submission}

The corresponding author is the guarantor of submission.

\section{Source of Support}

None

\section{Consent Statement}

Written informed consent was obtained from the patient for publication of this case report.

\section{Conflict of Interest}

Authors declare no conflict of interest.

\section{Copyright}

(C) 2018 Simona Delia Nicoară et al. This article is distributed under the terms of Creative Commons Attribution License which permits unrestricted use, distribution and reproduction in any medium provided the original author(s) and original publisher are properly credited. Please see the copyright policy on the journal website for more information.
Access full text article on other devices

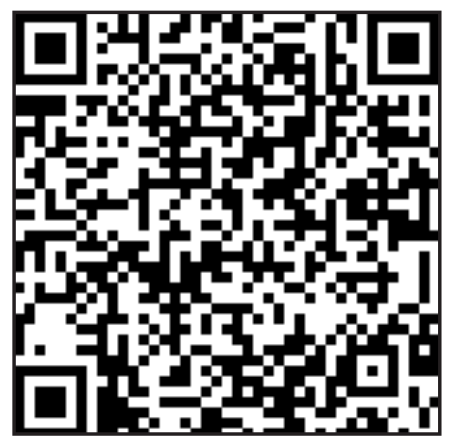

Access PDF of article on other devices

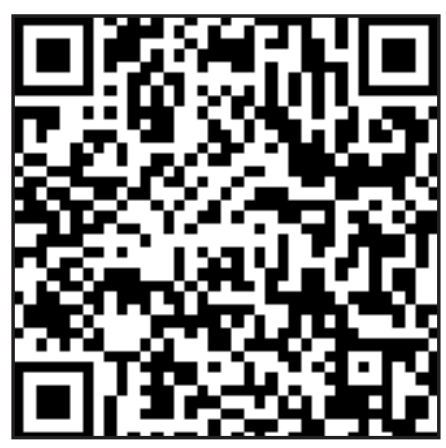

